\title{
Genome-wide silencing screen in mesothelioma cells reveals that loss of function of BAP1 induces chemoresistance to ribonucleotide reductase inhibition: implication for therapy
}

Agata Okonska ${ }^{1}$, Saskja Bühler ${ }^{1}$, Vasundhara Rao ${ }^{1}$, Manuel Ronner $^{1}$, Maxime Blijlevens ${ }^{2}$, Ida Van der Meulen-Muileman ${ }^{2}$, Renee de Menezes ${ }^{3}$, Egbert Smit ${ }^{4}$, Walter Weder ${ }^{5}$, Rolf Stahel $^{6}$, Lorenza Penengo ${ }^{7}$, Victor van Beusechem ${ }^{2}$, Emanuela Felley-Bosco ${ }^{1 *}$

${ }^{1}$ Laboratory of Molecular Oncology, Lungen- und Thoraxonkologie Zentrum, University Hospital Zürich, Sternwartstrasse 14, 8091 Zurich, Switzerland

${ }^{2}$ Department of Medical Oncology, VU University Medical Center, 1081 HV Amsterdam, the Netherlands

${ }^{3}$ Department of Epidemiology and Biostatistics, VU University Medical Center, 1081 HV Amsterdam, the Netherlands

${ }^{4}$ Department of Thoracic Surgery, NKI, Amsterdam, the Netherlands

${ }^{5}$ Division of Thoracic Surgery, University Hospital Zürich, R, 8091 Zurich, Switzerland

${ }^{6}$ Lungen- und Thoraxonkologie Zentrum, University Hospital Zürich, Ramistrasse 100, 8091 Zurich, Switzerland

${ }^{7}$ Institute of Molecular Cancer Research, University of Zürich, Zürich 8057, Switzerland

*Corresponding author: emanuela.felley-bosco@usz.ch, Phone + 4144255 2771, Fax +41 44255 5047

Keywords: BRCA1-associated protein-1, mesothelioma, gemcitabine, hydroxyurea, genotoxic stress 


\begin{abstract}
Introduction: Loss of function of BRCA1 associated protein 1 (BAP1) is observed in about $50 \%$ of malignant pleural mesothelioma (MPM) cases. The aim of this study was to investigate whether this aspect could be exploited for targeted therapy.
\end{abstract}

Methods: A genetically engineered model was established expressing either functional or nonfunctional BAP1 and whole-genome siRNA screens were performed assessing impaired survival between the two cell lines. Cytotoxity induced by gemcitabine and hydroxyurea were assessed in a panel of BAP1-WT and BAP1-mut/del cell lines. Functional studies were carried out in BAP1 mut/del cell line reconstituted with BAP1 WT or BAP1 C91A (catalytically dead mutant) and in BAP1 WT cell line upon siRNA-mediated knock-down of BAP1.

Results: The whole-genome siRNA screen unexpectedly revealed 11 hits $(\mathrm{FDR}<0.05)$ more cytotoxic for BAP1-proficient cells. Two actionable targets, RRM1 and RRM2, were validated and their inhibition mediated by gemcitabine or hydroxyurea respectively, was more cytotoxic in BAP1proficient cell lines. Upregulation of RRM2 upon gemcitabine and hydroxyurea was more profound in BAP1 mut/del cell lines. Increased lethality mediated by gemcitabine and hydroxyurea was observed in NCI-H2452 cells reconstituted with BAP1 WT but not with C91A mutant and upregulation of RRM2 in NCI-H2452-BAP1 WT spheroids was modest compared to control or C91A mutant. Finally, the opposite was observed after BAP1 knockdown in BAP1-proficient SPC111 cell line.

Conclusion: We found that BAP1 is involved in the regulation of RRM2 levels during replication stress. These observations reveal a potential therapeutic approach where MPM patients to be stratified depending on BAP status for gemcitabine treatment. 


\section{Introduction}

Malignant pleura mesothelioma (MPM) is an aggressive cancer deriving from the mesothelium and more frequently occurring in the pleural cavity. Because clinical symptoms only appear with advanced disease, and mesothelioma is characterized by being highly aggressive, the median survival is approximately 8 to 27 months depending on histotype and therapy ${ }^{1}$. Thus, a significant need for new therapeutic approaches is warranted.

In the era of personalized medicine, one strategy is to investigate weaknesses that are dependent on mutated genes, therefore our original intention was to investigate synthetic lethality with mutated BRCA1-associated protein (BAP1). Indeed, BAP1 is the second most mutated gene in MPM (COSMIC, cancer.sanger.ac.uk V85) after CDKN2A (cyclin-dependent kinase inhibitor 2A) and loss of function of BAP1 has been reported in up to $50 \%$ of $\mathrm{MPM}^{2},{ }^{3}$.

BAP1 belongs to the group of deubiquitinating enzymes whose main function is the removal of ubiquitin entities from different targets, thereby opposing the function of E3 ligases ${ }^{4.5} ;{ }^{6}$. BAP1 is found in multiprotein complexes and it takes part in several cellular processes including gene expression regulation ${ }^{7}$. For example, BAP1 dimer is found to form two different complexes with the chromatin binders ASXL1 and ASXL2, human homologs of Drosophila additional sex combs (ASX), which are both able to deubiquitinate histone monoubiquitylated (H2Aub1) ${ }^{8} ;{ }^{9}{ }^{10}$. H2Aub1 is considered to be a key effector in transcriptional repression mediated by polycomb repressor 1 complex in target gene promoters although there are examples where repressive action of PRC1 is independent of H2Aub1 ${ }^{11}$. In addition, Bap1 homolog in Drosophila, Calypso, in a complex with ASX named Polycomb repressive deubiquitinase (PR-DUB), is responsible for repression of HOX genes by maintaining $\mathrm{H} 2 \mathrm{~A}$ deubiquitinated in embryo while increasing $\mathrm{HOX}$ expression in particular tissues ${ }^{8}$. Therefore, although H2Aub1 is largely used to monitor BAP1 activity, it has an unclear and fine-tuning role in the control of gene expression.

BAP1 binds BRCA1-associated RING domain 1 (BARD), modifies BRCA ubiquitination and BAP1deficient cells are sensitive to ionizing radiation and PARP inhibition ${ }^{12} ;^{13}$. In addition, we recently described that in the presence of wild-type BAP1 the expression of an alternative splice isoform of 
Bap1 (BAP1 $\Delta$ ) missing part of the catalytic domain, sensitizes to PARP inhibition, likely by competing with full length BAP1 for complex formation ${ }^{14}$.

In this study, using a genetically engineered model expressing either functional or non-functional BAP1 in the same genetic background we performed whole-genome siRNA screens assessing impaired survival comparing BAP1-proficient vs. BAP1-deficient MPM cells. Silencing ribonucleotide reductase subunits RRM1 and RRM2 conferred an increased lethality in BAP1proficient cells. This observation was confirmed in a large panel of BAP1-proficient cells, which were more sensitive to ribonucleotide reductase inhibition compared to BAP1-deficient cells and we show that this is linked to a repressor activity of BAP1.

\section{Material \& Methods}

\section{Reagents}

Dulbecco's Modified Eagle's Medium/Nutrient Mixture F12 Ham (DMEM-F12) medium and Penicillin/Streptomycin 100X stock solution were purchased from Sigma-Aldrich Chemie GmbH (Buchs, Switzerland). Trypsin-EDTA $0.25 \% 1 \mathrm{X}$ solution and OptiMEM medium were purchased from GIBCO (Life Technologies Europe, Zug, Switzerland). Fetal calf serum (FCS, CVFSVF00-01) was purchased from PAN Biotech (Aidenbach, Germany). Puromycin was obtained from AppliChem (Darmstadt, Germany). Olaparib (AZD2281, Ku-0059436) was purchased from Selleckchem (Houston, TX), gemcitabine was obtained from Lilly (Indianapolis, IN) and hydroxyurea from AppliChem (Darmstadt, Germany).

\section{Cell culture}

The human mesothelioma cell lines used in this study were obtained either from ATCC (Wesel, Germany): HEK293, NCI-H226, NCI-H2452, NCI-H2052; or from Riken BRC (Ibaraki, Japan): ACC-Meso-1 and ACC-Meso-4; or from European Collection of Cell Cultures (Salisbury, UK): Mero-82. The following cell lines were established in our laboratory: SPC111, ZL55 and were 
cultured as previously described ${ }^{15}$. HEK293 cells were grown in DMEM supplemented with $10 \%$ FCS and $1 \%$ Penicillin/Streptomycin. All the other cell lines were maintained in DMEM-F12 supplemented with $15 \%$ FCS and 1\% Penicillin/Streptomycin solution. Stably transfected cells were selected with puromycin. All cell lines were cultured at $37{ }^{\circ} \mathrm{C}$ in a humidified $5 \% \mathrm{CO}_{2}$ incubator.

\section{RNAi screen}

High-throughput screening (HTS) was performed as previously described ${ }^{16}$, using established automated liquid handling procedures. Three individual genome-wide screens were performed per cell line. Briefly, the siARRAY Human Genome library (Dharmacon, Thermo Fisher, Scientific, Lafayette, CO) comprising single-target pools of four distinct siRNAs was dispensed into 384-well plates (Greiner, Kremsmunster, Austria) at a concentration of 1.5 pmol in $10 \mu 1$ X siRNA buffer (Dharmacon). siGENOME Non-Targeting control pool\#1 and the siGENOME UBB SMARTpool siRNA (Dharmacon) were used as negative and positive control respectively.

Plates were stored at $-20^{\circ} \mathrm{C}$ until use. Then, DharmaFECT 1 transfection reagent (Dharmacon) diluted in OptiMEM (GIBCO) was added to the wells using a Multidrop Combi (Thermo Fisher Scientific) (final concentration of $0.009 \mu 1 /$ well; $0.012 \%$ ). Within two hours, 500 cells/well in a volume of $55 \mu 1$ of DMEM-F12 were plated using microFill cell dispenser (BioTek, Winooski, VT) into 384-well plates containing the complexes, resulting in a final siRNA concentration of $20 \mathrm{nM}$ and a final volume of $75 \mu 1$. After plating, cells were grown at normal cell culture conditions for 5 days, and then $6 \mu 1$ of the CellTiter-Blue (Promega, Madison, WI) was added into the wells. After $4 \mathrm{~h}$ incubation, $15 \mu 1$ of $6 \%$ SDS was added to stop the reaction and fluorescence was measured (540Ex/590Em).

The potency of the selected hits was validated in deconvolution experiments in non-automated setup: four distinct siRNAs for each gene were tested via viability assay and via western blotting (in order to assess protein knockdown efficiency and correlation with observed lethality) using exactly the same conditions and reagents as described above.

\section{RNAi screen analysis}


Raw fluorescence data were processed in R. Data were $\log 2$-transformed for all the screens and lethality scores were calculated relative to controls on plate according to the equation: lethality score $=$ (median of siRNA $A_{x}$ - median of siNonTargeting) / (median siUBB - median siNonTargeting). Quantile normalization of lethality scores was calculated using CellHTS2 R package. Identification of scores significantly different between cell lines was analysed by limma R package.

\section{RNA extraction, cDNA synthesis and q-PCR}

$0.5 \mu \mathrm{g}$ of total RNA was extracted from cells using RNeasy isolation kit (Qiagen, Hilden, Germany) and reverse-transcribed using the Quantitect Reverse Transcription Kit (Qiagen). Real-time qPCR was performed using QuantiTect SYBR Green PCR kit (Qiagen) and products were detected on a 7900HT Fast real-Time PCR system (Applied Biosystems, Thermo Fisher Scientific). Relative mRNA levels were determined by comparing the PCR cycle thresholds between cDNA of a specific gene and histone H3 ( $\Delta \mathrm{Ct}$ method) ${ }^{17}$. Primers used for qPCR are listed in Supplementary Table 1.

\section{Protein extraction and Western Blotting}

Total protein extracts were obtained by lysing the cells with hot Laemmli sample buffer $(60 \mathrm{mM}$ Tris- $\mathrm{Cl} \mathrm{pH}$ 6.8, $100 \mathrm{mM}$ DTT, 5\% glycerol, 1, 7\% SDS) and pressed few times through syringes $(26 \mathrm{G}){ }^{17}$. Spheroids were collected $48 \mathrm{~h}$ post-treatment. Protein concentration was determined using a Pierce ${ }^{\mathbf{T M}} 660 \mathrm{~nm}$ Protein Assay (Thermo Fisher Scientific). Core histone extracts were prepared by acidic extraction as previously described ${ }^{14}$ and their concentration was determined using Bradford protein assay. Proteins were prepared by adding $6 \mathrm{X}$ reducing Laemmli buffer and boiling for $5 \mathrm{~min}$ and a total of $5 \mu \mathrm{g}$ of total protein extracts or $0.5 \mu \mathrm{g}$ of purified histones were separated on denaturing $10 \%$ or $15 \%$ SDS-PAGE gels based on the target size and proteins were transferred onto PVDF transfer membranes $(0.45 \mu \mathrm{m}$, Perkin Elmer, Waltham, MA). Membranes were probed with the following primary antibodies: mouse anti-BAP1 (C4, sc-28283), goat anti-RRM2 (E-16, sc-10846), mouse anti-p53 (DO-1, sc-126), rabbit anti-E2F (C-20, sc-633) obtained from Santa Cruz (Dallas, TX); rabbit anti-Histone H2A (ab18255), rabbit anti-RRM1 (EPR8483, ab137114), rabbit anti- 
bioRxiv preprint doi: https://doi.org/10.1101/381533; this version posted July 31,2018 . The copyright holder for this preprint (which was not certified by peer review) is the author/funder, who has granted bioRxiv a license to display the preprint in perpetuity. It is made available under aCC-BY-NC-ND 4.0 International license.

phospho KAP1 (Ser824, ab70369) purchased from Abcam (Cambridge, UK), rabbit anti-phospho-p53 (Ser15, no.9284) and anti-Ubiquitin (P4D1, no. 3936) obtained from Cell Signaling (Danvers, MA), rabbit anti-phospho-BAP1 (Ser592, no. 93733), mouse anti- $\gamma$ H2AX (Ser139, JBW30, no. 05-636) purchased from Millipore (Burlington, MA), rabbit anti-H3 (Poly6019) obtained from BioLegend (San Diego, CA) and mouse anti- $\beta$-actin (C4, MP691002) purchased from MP Biomedicals (Santa Ana, CA). Membranes were then incubated with the following secondary antibody rabbit anti-mouse IgGHRP (no. A9004), goat anti-rabbit IgG-HRP (no. A0545), and rabbit anti-goat IgG-HRP (no. A5420) were obtained from Sigma Aldrich. The signals were detected by enhanced chemiluminescence (Clarity TM ECL Substrate, BioRad, Hercules, CA) and detected by Fusion Digital Imager (Vilber Lourmat, Marne-la-Vallée, France).

\section{BAP1 cloning, sequencing and transfection}

Human BAP1 cDNA amplified from MPM cell lines was subcloned into the EcoRI/NheI site of the pCI-puro vector, which contains a puromycin resistance gene ${ }^{14}$. To obtain specific nonsynonymous mutations within BAP1 gene or synonymous mutations allowing siRNA resistance the QuikChange II Site-Directed Mutagenesis kit (Stratagene, San Diego, CA) was used. All inserts were validated by sequencing and all primers are indicated in Supplementary Table 1. For isogenic BAP1 cell lines used in the screen NCI-H2452 cells were transfected with pCI-Puro_BAP1_WT (Addgene \#68365) using Lipofectamine 2000 reagent (Thermo Fisher Scientific) according to manufacturer's instruction. For further experiments, HEK293T and NCI-H2452 cells were transfected transiently or stably with either control empty vector, pCI-Puro_BAP1_WT (Addgene \#108439) or pCI-Puro_BAP1_C91A (Addgene \#108438) using the same method of transfection.

\section{RNA interference and drug treatment}

In order to down-regulate BAP1 expression, ON-TARGETplus SMARTpool or single siRNAs against BAP1 or siGENOME Non-Targeting siRNA pool \#1 as well as DharmaFECT 1 transfection reagent were obtained from Dharmacon. For RRM1 and RRM2 knockdown efficiency validation in MPM cell 
bioRxiv preprint doi: https://doi.org/10.1101/381533; this version posted July 31,2018 . The copyright holder for this preprint (which was

not certified by peer review) is the author/funder, who has granted bioRxiv a license to display the preprint in perpetuity. It is made available under aCC-BY-NC-ND 4.0 International license.

lines, pools of the two best distinct siRNAs against RRM1 (siRRM1 \#3 and \#4) and RRM2 (siRRM2 $\# 1$ and \#3) were used at the same concentration as in the screen. Briefly, siRNA dissolved in $1 \mathrm{X}$ siRNA buffer (Dharmacon) was combined with transfection reagent dissolved in OptiMEM (final concentration of $0.42 \mu \mathrm{l} /$ well per well; $0.042 \%$ ) and incubated for $20 \mathrm{~min}$. Then, cells resuspended in normal growth medium were added to the siRNA/DharmaFECT 1 mixture and seeded onto plates, allowing for a final siRNA concentration of $20 \mathrm{nM} .0 .5 \times 10^{5}$ cells (12-well plate) were plated for whole cell protein lysates as wells as RNA extraction. $24 \mathrm{~h}$ later, cells were treated with either gemcitabine or hydroxyurea (final concentration of $0.1 \mu \mathrm{M}$ and $0.2 \mu \mathrm{M}$ respectively) and $48 \mathrm{~h}$ later protein lysates were prepared.

\section{Clonogenic assay}

Colony formation assays were performed as follows: NCI-H2452 clonal cells were plated at density of 1000/well in 6-well plate and subjected to treatment with different concentrations of a specific drug after 1 and 5 days. After additional 5 days cells were stained with Crystal Violet and colonies were counted by eye.

\section{Spheroids formation and viability assay}

Spheroids formation assays were performed as previously described ${ }^{18}$. At day 4 after seeding the spheroids were treated continuously with the drugs $(0.01,0.1,1.0,10.0,100.0$, and $500 \mu \mathrm{M}$ gemcitabine or $0.1,0.5,2.0 \mathrm{mM}$ of hydroxyurea or remained untreated) for 6 days and viability was analyzed using the CellTiter-Glo Luminescent Cell Viability Assay (Promega), to determine the ATP content. Luminescence was acquired using GloMax 96 Microplate Luminometer (Promega). Each experiment was performed in triplicate.

\section{Statistical analysis}

Fluorescence data from the cell viability screen were log2-transformed, normalized to the negative control (siNon-Targeting) per plate, and synthetic lethality scores were calculated. 
bioRxiv preprint doi: https://doi.org/10.1101/381533; this version posted July 31,2018 . The copyright holder for this preprint (which was

not certified by peer review) is the author/funder, who has granted bioRxiv a license to display the preprint in perpetuity. It is made available under aCC-BY-NC-ND 4.0 International license.

Statistical analysis was performed using GraphPad Prism version 5.04. Differences with $p<0.05$ were considered significant.

\section{Results}

\section{Whole-genome RNAi screen reveals genetic vulnerabilities in BAP1 WT cell line}

In order to identify genes whose inhibition induces synthetic lethality specifically in BAP1 loss-offunction MPM cells, we generated isogenic BAP1-proficient and BAP-deficient cell lines by stably transfecting NCI-H2452 BAP1 $1^{\mathrm{A} 95 \mathrm{D} /-5,19}$ cell line with either a BAP1 wild-type (BAP1) expression vector or an empty vector (EV). Multiple BAP1-proficient independent clones were generated and characterized by H2Aub1 levels, as well as their response to olaparib in comparison to EV clonal cell lines. The selected BAP1 expressing clone was shown to exhibit similar growth characteristics as an EV clone (data not shown). In addition, BAP1 expression decreased H2Aub1 (Figure 1A) as well as conferred resistance to olaparib, especially visible at $5 \mu \mathrm{M}$ (Figure 1B), as we had previously observed using another cell line ${ }^{14}$.

The final conditions (cell number per well, amount of DharmaFECT 1, concentration of siRNAs, incubation time for the screen as well as incubation time for the CellTiter-Blue readout) for the automated HTS setup were then optimized on the two clonal cell lines.

Three independent genome-wide screens per cell line were performed. The work-flow (Supplementary Figure 1) began with dispensing siARRAY whole human genome library comprising single-target pools of four distinct siRNAs, as well as negative and positive control siRNAs into 384-well plates. After storage until use, the experiment started by adding transfection reagent and subsequently cells to the plates to allow reverse transfection. After 5 days, viability was measured by reading fluorescence and lethality score and significant hits were evaluated for BAP1-proficient vs -deficient lines (Supplementary Figure 2). Differential lethality was then calculated between the two cell lines (Figure 1C). 1775 genes were found to be significantly $(p<0.05)$ different when comparing BAP1proficient vs BAP1-deficient cell line. Consistent with clones' characterization, we observed 
a difference in lethality score of 0.12 and of 0.08 between BAP1-proficient vs -deficient line for siPARP1 and for siPARP2, respectively. However, effects on viability were poor compared to effects of olaparib, likely because the drug inhibits both enzymes. From the list of 1775 genes we first considered the 191 genes where a differential lethality score $\geq 0.2$ between BAP1-proficient vs deficient line had been calculated. We searched for functional enrichment by gene ontology analysis using DAVID ${ }^{20}$. Interestingly, using the Functional Annotation Clustering tool, the most enriched functional cluster was a group of terms associated with RNA splicing and processing (enrichment score 4.23; Supplementary Table 2), which also had the highest score in a screen for genes involved in the so called replicative stress ${ }^{21}$, which is a term describing replication forks slowing or stalling by endogenously- or exogenously-derived impediments of DNA polymerases ${ }^{22}$. We then took into account only genes with a differential lethality score $\geq 0.3$ and FDR $<0.05$. The analysis revealed 11 significant differentially lethal genes (Table 1). Surprisingly, depletion of all the 11 hits were more cytotoxic for BAP1 WT cell line.

\section{BAP1-proficient cells are more sensitive to RRM1 and RRM2 silencing}

Two genes out of the 11 hits, namely RRM1 and RRM2, encode the two subunits that together form a protein heterotetramer (containing two copies of the catalytic subunit RRM1 and two copies of the regulatory subunit, RRM2) called ribonucleotide reductase (RNR), a key enzyme in de novo synthesis of dNTPs that converts ribonucleotide diphosphates (NDP) into deoxyribonucleotide diphosphates (dNDP), after which the NDP-kinase (NDPK) catalyzes the conversion of dNDPs to dNTPs ${ }^{23}$. Since RNR is of particular clinical relevance, as a drug targeting this enzyme is already used in second line therapy in mesothelioma, we decided to focus on RRM1 and RRM2 for further characterization. The genome-wide RNAi library comprises pools of four individual siRNAs per gene. Therefore, we deconvoluted each siRNA pool used in the screen for these 2 hits in order to exclude possible false-positive effects ${ }^{24}$. We investigated the effect of individual siRNAs on overall lethality as well as a BAP1-dependent lethality. Viability assays were performed with four individual siRNAs targeting either RRM1 (siRRM1 \#1, \#2, \#3, \#4) or RRM2 (siRRM2 \#1, \#2, \#3, \#4) (Figure 2A). 
Efficiency of RRM1 and RRM2 knockdown demonstrated that best knockdown was obtained with RRM1 \#3 and \#4 and RRM2 \#1 and \#3 (Supplementary Figure 3A and B). Moreover, as observed previously in the original screens, the BAP1 WT expressing clonal cell line was significantly more sensitive to RRM1 or RRM2 depletion compared to the EV clonal cell line (Figure 2A).

We then aimed at verifying the effect of RRM1 or RRM2 depletion in a broader panel of malignant pleural mesothelioma (MPM) cell lines. Four cell lines representing BAP1 wild-type group (BAP1 WT) (SPC111, ACC-Meso-1, NCI-H2052 and Mero82) $)^{14}$ and three BAP1 mutated or deleted (mut/del) cell lines (NCI-H226 ${ }^{25}$, NCI-H2452, ACC-Meso-4 ${ }^{26}$ ) were transfected with a pool of the two siRNAs targeting RRM1 or RRM2 mentioned above, and the efficiency of the knockdown in all cell lines was assessed (Supplementary Figure 4A and B). Interestingly we noticed that silencing either RRM1 or RRM2 upregulates the expression of the other subunit (Supplementary Figure 4A and B) in all cell lines, consistent with a reciprocal co-regulation ${ }^{27}$. We then assessed viability upon silencing. MPM cell lines expressing BAP1 WT demonstrated lower surviving fraction upon RRM1 and RRM2 knockdown compared to cells with BAP1 mut/del status (Figure 2B), suggesting higher sensitivity to siRNA-mediated depletion of RRM1 and RRM2. Noteworthy, expression of RRM1 as well as RRM2 was generally lower in BAP1 WT positive MPM cell lines on both mRNA and protein level compared to the BAP1 mut/del MPM cell lines (Supplementary Figure 5A and B), although this was not particularly associated with different growth rates. Publicly available TCGA mRNA expression data of 87 MPM samples (MESO) revealed that a similar inverse relationship between BAP1 and RRM1 or RRM2 exists as well in clinical samples (Supplementary Figure 6).

Overall, these data provide evidence that sensitivity of MPM cells to RRM1 or RRM2 depletion might depend on BAP1 status, BAP1 WT cell lines being more vulnerable.

\section{BAP1 WT MPM cell lines are more sensitive to gemcitabine and hydroxyurea}

As there are known selective inhibitors against RRM1 and RRM2 already used in clinics, namely gemcitabine and hydroxyurea, respectively, we aimed to test their effect on growth on a panel of MPM cell lines. For that reason, BAP1 WT and BAP1 mut/del cell lines were grown in spheroids to better 
mimic in vivo conditions ${ }^{18}$ and subsequently treated with increasing concentrations of gemcitabine $(0.01,0.1,1.0,10,100,500 \mu \mathrm{M})$ or hydroxyurea $(0.1,0.5,2.0 \mathrm{mM})$. Consistent with the effect of RRM1i, gemcitabine was approximately thousand fold more potent in decreasing the viability in the BAP1 WT group (Figure 3A and C). A similar effect was observed in spheroids treated with hydroxyurea, where the most evident separation in lethality between the two groups was detected at the concentration of $2 \mathrm{mM}$ (Figure 3B and C).

Taken together, these data provide evidence and confirmation of the previous findings based on siRNA interference, that BAP1 WT positive MPM cells are more sensitive to RRM1 and RRM2 inhibition.

\section{Expression of BAP1 sensitizes NCI-2452 cells to RNR inhibition}

In order to understand whether decreased expression of RRM1 and RRM2 observed in 2D conditions was maintained in $3 \mathrm{D}$, thereby potentially underlying the differential sensitivity to RNR inhibition, we assessed RRM1 and RRM2 expression in baseline and upon drug treatment.

Since the previous experiments revealed certain vulnerabilities in BAP1 WT MPM cell lines, we decided to investigate whether BAP1 was an underlying genetic factor determining this susceptibility. Contrarily to the observation in 2D, in 3D there was no obvious differential expression of RRM1 and RRM2 between BAP1 WT and BAP1 mut/del group under basal conditions (Figure 4A). In addition, we observed no clear differential pattern in RRM1 expression upon treatment (Figure 4A, left panel), most likely due to the induction of ubiquitination and degradation of RRM1 upon gemcitabine treatment ${ }^{28}$. However, expression of RRM2 was significantly more up-regulated upon gemcitabine and hydroxyurea treatment in BAP1 mut/del compared to BAP1 WT group (Figure 4A, right panel), possibly explaining their resistance to the treatment.

Although we did not measure cellular dNTP pools and previous studies showed no dNTP depletion upon hydroxyurea in mammalian cells ${ }^{29}$, we assume that gemcitabine caused nucleotide depletion because supplementation of dNMP rescued its toxicity (data not shown). Decreasing the deoxynucleotide pool leads to slowing or stalling of the replication forks and loss of polymerase 
processivity leading to formation of a tract of single stranded DNA causing genetic instability resulting in $\mathrm{H} 2 \mathrm{AX}$ phosphorylation ${ }^{30-32}$. Therefore, we tested expression of $\mathrm{yH} 2 \mathrm{AX}$, as well as pKAP1, two down-stream targets of ATM, and therefore being indicative of replicative stress. $\mathrm{yH} 2 \mathrm{AX}$ levels were higher in the treated samples (Figure 4B). Similarly, pKAP1 levels were up-regulated upon both gemcitabine and hydroxyurea treatment. P53 was phosphorylated and stabilized upon gemcitabine and hydroxyurea treatment, indicating replicative stress in concordance with $\mathrm{yH} 2 \mathrm{AX}$ levels (Figure 4C). We observed no p53 signal in NCI-H2452 cell line, which has a mutation inducing a truncated $\mathrm{p} 53^{33}$, and very low levels in ACC-Meso-1 and ACC-Meso-4, which have WT p53 ${ }^{34}$.

Since BAP1 is phosphorylated in an ATM-dependent manner at S592 35, 36, we tested whether gemcitabine or hydroxyurea treatment had an impact on BAP1 S592 phosphorylation. As expected, pBAP1 was detectable in all BAP1 WT cell lines samples upon the treatments (Figure 4B). Noteworthy, although the pBAP1 fraction was clearly elevated upon the treatment compared to the untreated cells, the total BAP1 level diminished in both gemcitabine and hydroxyurea treated BAP1 WT spheroids.

Altogether, this data suggests that although DNA damage signalling was activated in both BAP1proficient and -deficient cells, BAP1 mut/del cell lines are characterized by higher up-regulation of RRM2 upon a treatment, which suggests a possible resistance mechanism of BAP1 mut/del cell lines to gemcitabine and hydroxyurea.

\section{Sensitization of MPM cells to gemcitabine and hydroxyurea is dependent on deubiquitinating activity of BAP1}

In order to exclude the possibility of the interplay of the diverse genetic backgrounds in all tested cell lines, we generated de novo NCI-H2452 cell line stably transfected with either EV, or BAP1 WT or a BAP1 C91A, a previously described catalytic dead mutant (C91A) ${ }^{25,37}$. Then, we monitored BAP1 expression at protein level (Supplementary Figure 7A) as well as deubiquitinating (DUB) activity of the two variants. Interestingly, endogenous mutated BAP1 is phosphorylated under basal 2D conditions, contrarily to what we had observed in basal conditions in 3D (see Figure 4B). HEK293 
cells transiently transfected with BAP1 WT show diminished levels of H2Aub1, in contrast to C91A mutant (Supplementary Figure 7B) consistent with what has been previously reported ${ }^{8,25,37 .}$ Subsequently, we tested the viability of the cells grown in spheroids upon gemcitabine or hydroxyurea treatment.

As expected, cells expressing BAP1 WT, but not C91A mutant, are sensitized to gemcitabine compared to cells transfected with EV (Figure 5A). The same observation was made for cells treated with hydroxyurea (Figure 5B). These results suggest that BAP1 DUB activity plays a crucial role in the mechanism of the observed sensitization.

\section{BAP1 regulates $R R M 2$ up-regulation in replication stress}

To further investigate mechanisms underlying BAP1 effects on sensitization to gemcitabine and hydroxyurea we tested whether BAP1 WT reconstitution in NCI-H2452 cells would rescue these cells from high RRM2 up-regulation. NCI-H2452 cell lines stably expressing either EV, or BAP1 WT or C91A mutant were grown in 3D and treated with gemcitabine or hydroxyurea for $48 \mathrm{~h}$. NCI-H2452 cell line reconstituted with BAP1 WT but not C91A mutant, showed a decreased induction of RRM2 expression compared to EV-transfected cells upon the treatments (Figure 6A). Conversely, silencing BAP1 in SPC111 cells expressing BAP1 WT resulted in a significant increased expression of RRM2 on protein as well as on mRNA level upon treatment of the cells with gemcitabine or hydroxyurea (Figure 6B). DNA upregulates RRM2 at least partially via upregulation of E2F1 transcription factor ${ }^{38}$, therefore we investigated expression of the latter and observed indeed increased levels of E2F1 upon drug treatment in BAP1 knocked down cells.

As we had observed in $3 \mathrm{D}$, drug treatment led to a decrease of BAP1 protein, but levels of mRNA were not affected (Figure 6B).

Altogether, these data provide further evidence that BAP1 mut/del cells react to replication stressinducing agents with higher RRM2 up-regulation compared to BAP1 WT cells, suggesting an involvement of BAP1 WT in modulating E2F1 and RRM2 increase in replicative stress conditions (Supplementary Figure 8). 


\section{Discussion}

In this study we describe that BAP1 loss induces chemoresistance to drugs inhibiting RNR in mesothelioma cells and this observation has immediate clinical implications since gemcitabine is used in second line mesothelioma treatment ${ }^{39}$.

Differential synthetic lethality between BAP1-proficient vs. -deficient cells included the RNR subunits RRM1 and RRM2 and we concentrated on understanding the underlying mechanisms because of the translational importance of this observation. RNR activity is necessary for DNA replication and repair. The activity of this enzyme is controlled at the transcriptional level during the cell cycle with maximal levels during S-phase. While levels of RRM1 protein are almost constant in proliferating cells owing to a long half-life, the RRM2 protein is specifically degraded in late mitosis after polyubiquitination by the anaphase-promoting complex-Cdh1 ubiquitin ligase (reviewed in ${ }^{40}$ ).

It has been estimated that in $3 \mathrm{D}$ spheroids about one third of the cells are in quiescent state ${ }^{41}$, corresponding better to the proliferation status of tumoral cells ${ }^{42}$ compared to $2 \mathrm{D}$ cell culture. Therefore, in this model there are proliferating cells and non-proliferating cells, which could be less sensitive to the lack of deoxynucleotides. Nevertheless, using this system we observed more than $3 \log$ differences for the IC50 between BAP1-proficient and -deficient cells. Quiescent cells still need deoxynucleotides for DNA repair and mitochondrial DNA synthesis, and a certain threshold concentration is necessary for some repair DNA polymerases (reviewed in ${ }^{40}$ ). Therefore, cells also express an alternative subunit having the same properties as RRM2, i.e., RR2B, which allows cells to produce enough deoxynucleotides in the absence of RRM2 ${ }^{29}$. However, levels of RR2B were very low even in the 3D model under conditions of RRM1 and RRM2 inhibition (data not shown), excluding any compensatory role for RR2B under our experimental conditions.

Consistent with a previous study where silencing RRM1 and RRM2 caused genomic instability detectable through phosphorylation of histone variant $\mathrm{H} 2 \mathrm{AX}{ }^{21}$, we observed activation of ATM in spheroids upon gemcitabine and hydroxyurea treatment. As expected, this led also to BAP1 phosphorylation since BAP1 is phosphorylated upon DNA damage on ATM and ATR consensus sites 36, 43 . Ionizing radiation (IR) or hydroxyurea result in rapid phosphorylation of a small fraction of 
BAP1 at S592 in S-phase and dissociation from chromatin, to presumably regulate expression of DNA damage repair genes ${ }^{35}$. In parallel to BAP1 phosphorylation we observed a decrease of BAP1 protein levels, consistent with a previous study where Bap1 levels decreased after IR ${ }^{44}$. Upon gemcitabine and hydroxyurea treatment we could not detect any significant downregulation of BAP1 mRNA, while BAP1 protein levels were maintained in cells transfected with a WT BAP1 expression plasmid (data not shown). Therefore, we hypothesize that downregulation of BAP1 under conditions of genomic instability occurs at a post-transcriptional level, possibly by targeting the BAP1 3'UTR, which could be further investigated.

We observed a repressor role for BAP1 on the expression of RRM2 upon the response to inhibition of RNR, when RRM1 and RRM2 are upregulated. It is likely that the identification of RRM1 and RRM2 as BAP1 synthetic lethal targets in the screen, which was performed in the absence of any exogenously induced replicative stress, was due to the serendipitous choice of the model, where we sought differences between BAP1-proficient vs.-deficient cells in transfected cells that undergo replicative stress and BAP1 phosphorylation when grown in 2D.

Upregulation of RRM1 and RRM2 is consistent with various studies showing that DNA damaging agents increase the levels of RNR subunits ${ }^{38,45,46}$. In mammalian cells, upregulation of RRM2 after exposure of the cells to HU has been linked to decreased binding of RFX repressor to RRM2 promoter ${ }^{47}$. Therefore a possible scenario is that RFX is more freely released in the absence of BAP1. Although RFX is not among previously reported BAP1 interactors ${ }^{48}$, it might have been missed because this wide interactome study had been performed in the absence of replicative stress.

Another possibility is that BAP1 interacts with positive regulators of RRM2. RRM1 and RRM2 are part of the genes upregulated in Retinoblastoma $(\mathrm{Rb})$ deficient mouse embryo fibroblast ${ }^{49}$. $\mathrm{Rb}$ negatively controls the activity of $\mathrm{E} 2 \mathrm{~F}$ transcription factors, therefore regulators of $\mathrm{E} 2 \mathrm{~F}$ transcription factors can affect RRM1 and RRM2 expression. Genotoxic stress upregulates RRM2 at least partially via upregulation of E2F $1{ }^{38}$. The latter is stabilized downstream of ATM activity ${ }^{50}$ and we also observed increased levels of E2F1 upon silencing of BAP1 in replicative stress condition. E2F1 is regulated by post-translational modifications during the cell cycle progression and in response 
to DNA damage (reviewed ${ }^{51}$ ) including by K63 ubiquitination ${ }^{52,53}$ and UCH37, a member of the same DUB family as BAP1, has been shown to increase E2F1 activity ${ }^{52}$. In addition, BAP1 is known to bind Host Cell factor 1 (HCF-1) and the latter recruits activating methyltransferases to E2Fresponsive promoters resulting in transcriptional activation of cell-cycle specific genes ${ }^{54}$. However, although BAP1 deubiquitinates HCF-1 on K48-linked ubiquitin chains ${ }^{55,56}$, differential gene expression revealed a significant BAP1-dependent effect on RRM1 but not RRM2 upregulation ${ }^{57}$. This is in contrast to the inverse relationship that we have observed, however, BAP1 is a complex protein acting as gene expression activator or repressor depending on the context. Even within the same cells, BAP1 leads to activation or repression of different FOXK2 target genes after forming a complex with FOXK2 ${ }^{58}$. The question whether BAP1 would have any effect on RRM1 and RRM2 via interaction with HCF-1 upon replicative stress conditions remains open.

Finally, BRCA1 acts as a transcriptional co-activator of RRM2 ${ }^{59}$, so BAP1 effects could be mediated by its interaction with BRCA1. However, as this mechanism of RRM2 regulation could be observed in glioblastoma cells but not in other cancer cell type, it is less likely.

BAP1 suppression of RRM2 expression upon replicative stress is consistent with tumor suppressor activity of BAP1 since overexpression of RRM2 is mutagenic in mouse cells and promotes lung carcinogenesis ${ }^{60}$. Interestingly, high levels of RRM1 and RRM2 expression are associated with worst overall survival in MPM patients (Supplementary Figure 9) and it would be of interest to investigate whether this is associated with BAP1 status. BAP1 could be part of the tightly regulated mechanisms to keep RRM2 expression under control since BAP1 is downregulated and phosphorylated upon replicative stress and phosphorylation has been linked to dissociation from chromatin (see above).

As previously mentioned, gemcitabine is already part of second line treatment of MPM patients and the investigation of response according to BAP1 status will be assessed in current clinical trials (NCT02991482, EORTC-NAVALT19) to verify whether BAP1 status is a predictor of response to this therapy.

\section{Acknowledgement}


This work was supported by the Walter Bruckerhoff Stiftung, the Swiss National Science Foundation grant CRSII3 147697/1 and the Stiftung für Angewandte Krebsforchung. We also would like to thank Franziska Pfistner for assistance with the side-directed mutagenesis protocol.

\section{References}

1. Yap TA, Aerts JG, Popat S, et al. Novel insights into mesothelioma biology and implications for therapy. Nat Rev Cancer 2017;17:475-488.

2. Wang A, Papneja A, Hyrcza M, et al. Gene of the month: BAP1. J Clin Pathol 2016;69:750753.

3. Farzin M, Toon CW, Clarkson A, et al. Loss of expression of BAP1 predicts longer survival in mesothelioma. Pathology 2015;47:302-307.

4. Jensen DE, Proctor M, Marquis ST, et al. BAP1: a novel ubiquitin hydrolase which binds to the BRCA1 RING finger and enhances BRCA1-mediated cell growth suppression. Oncogene 1998;16:1097-1112.

5. Bott $\mathrm{M}$, Brevet $\mathrm{M}$, Taylor $\mathrm{BS}$, et al. The nuclear deubiquitinase BAP1 is commonly inactivated by somatic mutations and 3p21.1 losses in malignant pleural mesothelioma. Nat Genet 2011;43:668-672.

6. Komander D, Clague MJ, Urbe S. Breaking the chains: structure and function of the deubiquitinases. Nat Rev Mol Cell Biol 2009;10:550-563.

7. Carbone M, Yang H, Pass HI, et al. BAP1 and cancer. Nat Rev Cancer 2013;13:153-159.

8. Scheuermann JC, de Ayala Alonso AG, Oktaba K, et al. Histone H2A deubiquitinase activity of the Polycomb repressive complex PR-DUB. Nature 2010;465:243-247.

9. Daou S, Hammond-Martel I, Mashtalir N, et al. The BAP1/ASXL2 Histone H2A Deubiquitinase Complex Regulates Cell Proliferation and Is Disrupted in Cancer. J Biol Chem 2015;290:28643-28663. 
10. Sahtoe DD, van Dijk WJ, Ekkebus R, et al. BAP1/ASXL1 recruitment and activation for H2A deubiquitination. Nat Commun 2016;7:10292.

11. Illingworth RS, Moffat M, Mann AR, et al. The E3 ubiquitin ligase activity of RING1B is not essential for early mouse development. Genes Dev 2015;29:1897-1902.

12. Pena-Llopis S, Vega-Rubin-de-Celis S, Liao A, et al. BAP1 loss defines a new class of renal cell carcinoma. Nat Genet 2012;44:751-759.

13. Yu H, Pak H, Hammond-Martel I, et al. Tumor suppressor and deubiquitinase BAP1 promotes DNA double-strand break repair. Proc Natl Acad Sci U S A 2014;111:285-290.

14. Parrotta R, Okonska A, Ronner M, et al. A Novel BRCA1-Associated Protein-1 Isoform Affects Response of Mesothelioma Cells to Drugs Impairing BRCA1-Mediated DNA Repair. J Thorac Oncol 2017.

15. Thurneysen C, Opitz I, Kurtz S, et al. Functional inactivation of NF2/merlin in human mesothelioma. Lung Cancer 2009;64:140-147.

16. de Lange J, Faramarz A, Oostra AB, et al. Defective sister chromatid cohesion is synthetically lethal with impaired APC/C function. Nat Commun 2015;6:8399.

17. Kresoja-Rakic J, Kapaklikaya E, Ziltener G, et al. Identification of cis- and trans-acting elements regulating calretinin expression in mesothelioma cells. Oncotarget 2016;7:21272-21286.

18. Echeverry N, Ziltener G, Barbone D, et al. Inhibition of autophagy sensitizes malignant pleural mesothelioma cells to dual PI3K/mTOR inhibitors. Cell Death Dis 2015;6:e1757.

19. Yoshikawa $\mathrm{Y}$, Sato A, Tsujimura $\mathrm{T}$, et al. Frequent inactivation of the BAP1 gene in epithelioid-type malignant mesothelioma. Cancer Sci 2012;103:868-874.

20. Huang da W, Sherman BT, Lempicki RA. Systematic and integrative analysis of large gene lists using DAVID bioinformatics resources. Nat Protoc 2009;4:44-57.

21. Paulsen RD, Soni DV, Wollman R, et al. A genome-wide siRNA screen reveals diverse cellular processes and pathways that mediate genome stability. Molecular cell 2009;35:228-239.

22. Zeman MK, Cimprich KA. Causes and consequences of replication stress. Nat Cell Biol 2014;16:2-9. 
23. Aye Y, Li M, Long MJ, et al. Ribonucleotide reductase and cancer: biological mechanisms and targeted therapies. Oncogene 2015;34:2011-2021.

24. Echeverri CJ, Beachy PA, Baum B, et al. Minimizing the risk of reporting false positives in large-scale RNAi screens. Nature methods 2006;3:777-779.

25. Ventii KH, Devi NS, Friedrich KL, et al. BRCA1-associated protein-1 is a tumor suppressor that requires deubiquitinating activity and nuclear localization. Cancer Res 2008;68:6953-6962.

26. Hakiri S, Osada H, Ishiguro F, et al. Functional differences between wild-type and mutanttype BAP1 tumor suppressor against malignant mesothelioma cells. Cancer Sci 2015.

27. Sagawa M, Ohguchi H, Harada T, et al. Ribonucleotide Reductase Catalytic Subunit M1 (RRM1) as a Novel Therapeutic Target in Multiple Myeloma. Clin Cancer Res 2017;23:5225-5237.

28. Zhang Y, Li X, Chen Z, et al. Ubiquitination and degradation of ribonucleotide reductase M1 by the polycomb group proteins RNF2 and Bmi1 and cellular response to gemcitabine. PLoS One 2014;9:e91186.

29. Hakansson P, Hofer A, Thelander L. Regulation of mammalian ribonucleotide reduction and dNTP pools after DNA damage and in resting cells. J Biol Chem 2006;281:7834-7841.

30. Ewald B, Sampath D, Plunkett W. H2AX phosphorylation marks gemcitabine-induced stalled replication forks and their collapse upon S-phase checkpoint abrogation. Mol Cancer Ther 2007;6:1239-1248.

31. Burma S, Chen BP, Murphy M, et al. ATM phosphorylates histone H2AX in response to DNA double-strand breaks. J Biol Chem 2001;276:42462-42467.

32. Papadopoulou C, Guilbaud G, Schiavone D, et al. Nucleotide Pool Depletion Induces GQuadruplex-Dependent Perturbation of Gene Expression. Cell Rep 2015;13:2491-2503.

33. Manfredi JJ, Dong J, Liu WJ, et al. Evidence against a role for SV40 in human mesothelioma. Cancer Res 2005;65:2602-2609.

34. Usami N, Fukui T, Kondo M, et al. Establishment and characterization of four malignant pleural mesothelioma cell lines from Japanese patients. Cancer Sci 2006;97:387-394. 
35. Eletr ZM, Yin L, Wilkinson KD. BAP1 is phosphorylated at serine 592 in S-phase following DNA damage. FEBS Lett 2013;587:3906-3911.

36. Stokes MP, Rush J, Macneill J, et al. Profiling of UV-induced ATM/ATR signaling pathways. Proc Natl Acad Sci U S A 2007;104:19855-19860.

37. Peng H, Prokop J, Karar J, et al. Familial and Somatic BAP1 Mutations Inactivate ASXL1/2Mediated Allosteric Regulation of BAP1 Deubiquitinase by Targeting Multiple Independent Domains. Cancer Res 2018;78:1200-1213.

38. Zhang YW, Jones TL, Martin SE, et al. Implication of checkpoint kinase-dependent upregulation of ribonucleotide reductase R2 in DNA damage response. J Biol Chem 2009;284:1808518095.

39. McCambridge AJ, Napolitano A, Mansfield AS, et al. Progress in the Management of Malignant Pleural Mesothelioma in 2017. J Thorac Oncol 2018;13:606-623.

40. Thelander L. Ribonucleotide reductase and mitochondrial DNA synthesis. Nat Genet 2007;39:703-704.

41. Daubriac J, Fleury-Feith J, Kheuang L, et al. Malignant pleural mesothelioma cells resist anoikis as quiescent pluricellular aggregates. Cell Death Differ 2009;16:1146-1155.

42. Kadota K, Suzuki K, Colovos C, et al. A nuclear grading system is a strong predictor of survival in epitheloid diffuse malignant pleural mesothelioma. Mod Pathol 2012;25:260-271.

43. Matsuoka S, Ballif BA, Smogorzewska A, et al. ATM and ATR substrate analysis reveals extensive protein networks responsive to DNA damage. Science 2007;316:1160-1166.

44. Ofir R, Zhang LC, Kyne AP, et al. Gamma-radiation-induced growth arrest and apoptosis in p53-null lymphoma cells is accompanied by modest transcriptional changes in many genes. DNA and cell biology 2000;19:29-37.

45. Lin ZP, Belcourt MF, Cory JG, et al. Stable suppression of the R2 subunit of ribonucleotide reductase by R2-targeted short interference RNA sensitizes p53(-/-) HCT-116 colon cancer cells to DNA-damaging agents and ribonucleotide reductase inhibitors. J Biol Chem 2004;279:27030-27038. 
46. D'Angiolella V, Donato V, Forrester FM, et al. Cyclin F-mediated degradation of ribonucleotide reductase M2 controls genome integrity and DNA repair. Cell 2012;149:1023-1034.

47. Lubelsky Y, Reuven N, Shaul Y. Autorepression of $\mathrm{rfx} 1$ gene expression: functional conservation from yeast to humans in response to DNA replication arrest. Mol Cell Biol 2005;25:10665-10673.

48. Sowa ME, Bennett EJ, Gygi SP, et al. Defining the human deubiquitinating enzyme interaction landscape. Cell 2009;138:389-403.

49. Markey MP, Bergseid J, Bosco EE, et al. Loss of the retinoblastoma tumor suppressor: differential action on transcriptional programs related to cell cycle control and immune function. Oncogene 2007;26:6307-6318.

50. Lin WC, Lin FT, Nevins JR. Selective induction of E2F1 in response to DNA damage, mediated by ATM-dependent phosphorylation. Genes Dev 2001;15:1833-1844.

51. Biswas AK, Johnson DG. Transcriptional and nontranscriptional functions of E2F1 in response to DNA damage. Cancer Res 2012;72:13-17.

52. Mahanic CS, Budhavarapu V, Graves JD, et al. Regulation of E2 promoter binding factor 1 (E2F1) transcriptional activity through a deubiquitinating enzyme, UCH37. J Biol Chem 2015;290:26508-26522.

53. Glorian V, Allegre $\mathrm{J}$, Berthelet $\mathrm{J}$, et al. DNA damage and $\mathrm{S}$ phase-dependent E2F1 stabilization requires the cIAP1 E3-ubiquitin ligase and is associated with K63-poly-ubiquitination on lysine 161/164 residues. Cell Death Dis 2017;8:e2816.

54. Tyagi $\mathrm{S}$, Chabes AL, Wysocka J, et al. E2F activation of $\mathrm{S}$ phase promoters via association with HCF-1 and the MLL family of histone H3K4 methyltransferases. Molecular cell 2007;27:107119.

55. Machida YJ, Machida Y, Vashisht AA, et al. The deubiquitinating enzyme BAP1 regulates cell growth via interaction with HCF-1. J Biol Chem 2009;284:34179-34188. 
56. Misaghi S, Ottosen S, Izrael-Tomasevic A, et al. Association of C-terminal ubiquitin hydrolase BRCA1-associated protein 1 with cell cycle regulator host cell factor 1. Mol Cell Biol 2009;29:2181-2192.

57. Yu H, Mashtalir N, Daou S, et al. The ubiquitin carboxyl hydrolase BAP1 forms a ternary complex with YY1 and HCF-1 and is a critical regulator of gene expression. Mol Cell Biol 2010;30:5071-5085.

58. Okino Y, Machida Y, Frankland-Searby S, et al. BRCA1-associated protein 1 (BAP1) deubiquitinase antagonizes the ubiquitin-mediated activation of FoxK2 target genes. J Biol Chem 2015;290:1580-1591.

59. Rasmussen RD, Gajjar MK, Tuckova L, et al. BRCA1-regulated RRM2 expression protects glioblastoma cells from endogenous replication stress and promotes tumorigenicity. Nat Commun $2016 ; 7: 13398$.

60. Xu X, Page JL, Surtees JA, et al. Broad overexpression of ribonucleotide reductase genes in mice specifically induces lung neoplasms. Cancer Res 2008;68:2652-2660. 
Table 1. Candidate genes with differential lethality in the two cell lines meeting the following criteria: differential lethality score $\geq 0.3$ and $F D R<0.05$.

\begin{tabular}{lllrr} 
Gene & Full gene name & Accession & p-value & FDR \\
\hline CANX & Calnexin & NM_001746 & $1.82 \mathrm{E}-06$ & 0.020 \\
\hline ZBTB2 & Zinc Finger And BTB Domain Containing 2 & NM_020861 & $4.12 \mathrm{E}-06$ & 0.020 \\
\hline RRM2 & Ribonucleoside-diphosphate reductase subunit M2 & NM_001034 & $4.25 \mathrm{E}-06$ & 0.020 \\
\hline RB1CC1 & RB1 Inducible Coiled-Coil 1 & NM_014781 & $4.35 \mathrm{E}-06$ & 0.020 \\
\hline RFT1 & RFT1 Homolog & NM_052859 & $4.69 \mathrm{E}-06$ & 0.020 \\
\hline RBM8A & RNA Binding Motif Protein 8A & NM_005105 & $7.46 \mathrm{E}-06$ & 0.024 \\
\hline RAN & ras-related nuclear protein & NM_006325 & $7.60 \mathrm{E}-06$ & 0.024 \\
\hline SF3B6 & splicing factor 3b subunit 6 & NM_016047 & $1.01 \mathrm{E}-05$ & 0.025 \\
\hline RRM1 & Ribonucleotide Reductase Catalytic Subunit M1 & NM_001033 & $1.07 \mathrm{E}-05$ & 0.025 \\
\hline COL20A1 & Collagen Type XX Alpha 1 & NM_020882 & $1.15 \mathrm{E}-05$ & 0.025 \\
\hline
\end{tabular}


A

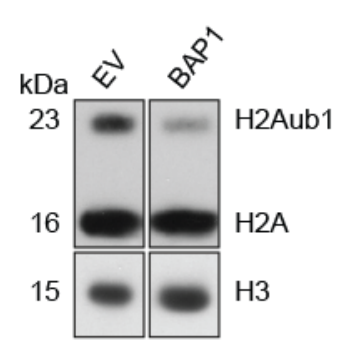

B

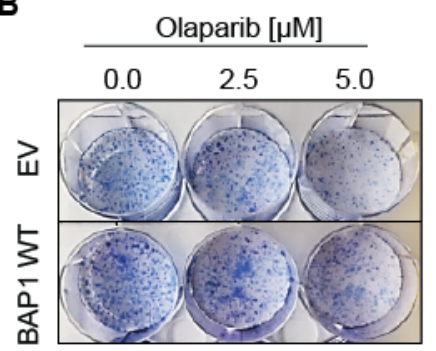

C

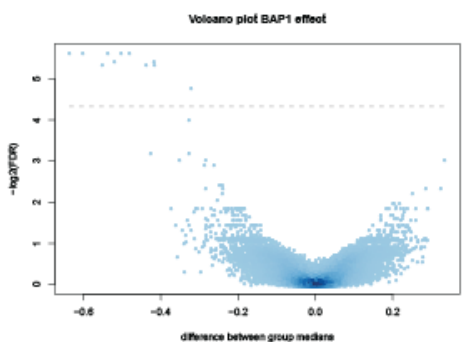

Figure 1. Generation of isogenic BAP1-proficient and BAP1-deficient cell lines and results of the genome-wide RNAi screen. (A) Anti-H2A and -H3 western blot performed on histone extracts obtained from clonal NCI-H2452 cell line transfected with either empty vector (EV) or BAP1 wildtype (BAP1). (B) Response to olaparib of clonal NCI-H2452 cell line transfected with either empty vector (EV) or wild-type BAP1 (BAP1) tested by clonogenic assay. (C) Volcano plot of lethality scores in BAP1-proficient vs BAP1-deficient clonal cell line. The $\mathrm{x}$-axis specifies the difference in lethality scores between the two groups and the y-axis specifies the negative logarithm to the base 2 of the FDR. Grey horizontal line reflects the filtering criteria (differential lethality score $\geq 0.3, p<0.05$ and FDR $<0.05)$. 
A

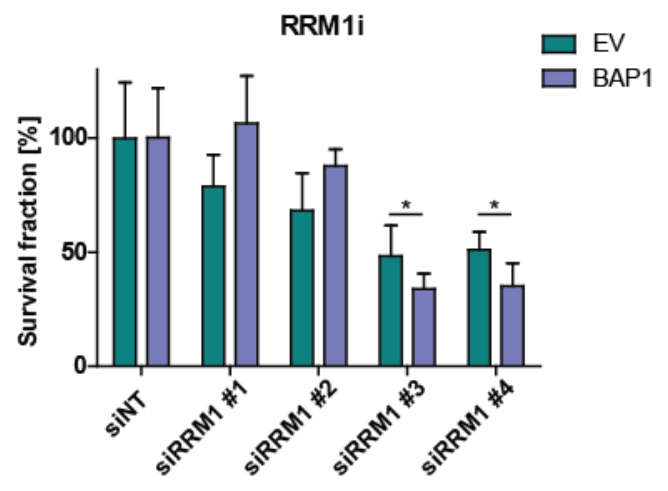

B

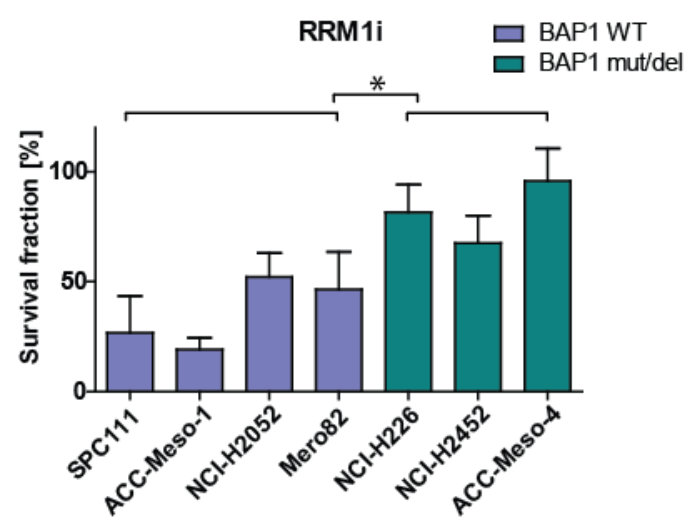

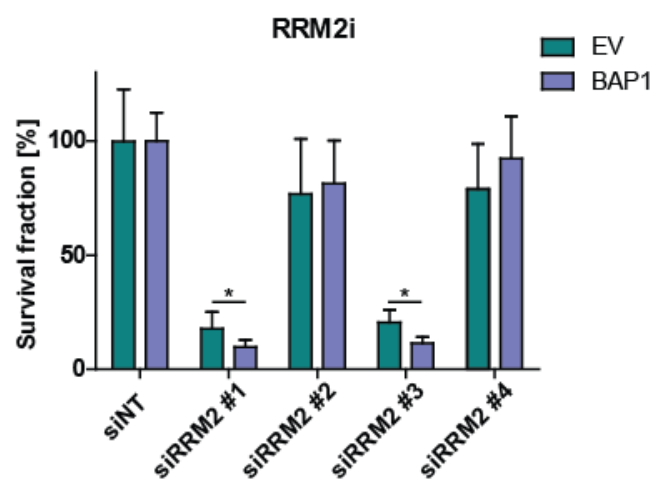

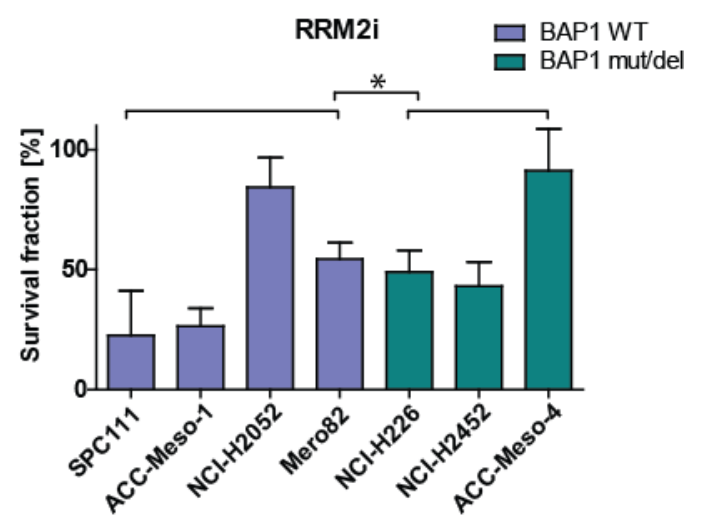

Figure 2. RRM1 and RRM2 knockdown is more lethal in BAP1 proficient cell lines. (A)

Deconvolution of siRRM1 and siRRM2 pools used in the screen performed on the same isogenic cell lines as in original screens. Single siRNAs targeting RRM1 were tested via viability assay. (B) Two best single siRNA for both RRM1 and RRM2 were pooled and tested on a panel of MPM cell lines via viability assay. BAP1 WT cell lines (NCI-H2052, ACC-Meso-1, Mero82, and SPC111) are represented in blue and BAP1 mut/del cell lines (ACC-Meso-4, NCI-H226, NCI-H2452) in green. Data shown are relative to the siNon-Targeting control (siNT). Significance was determined by MannWhitney U-test $\left({ }^{*} p<0.05\right)$. 
A

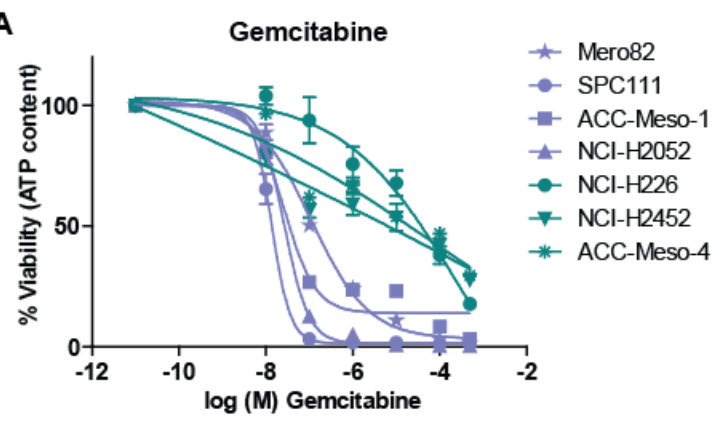

B
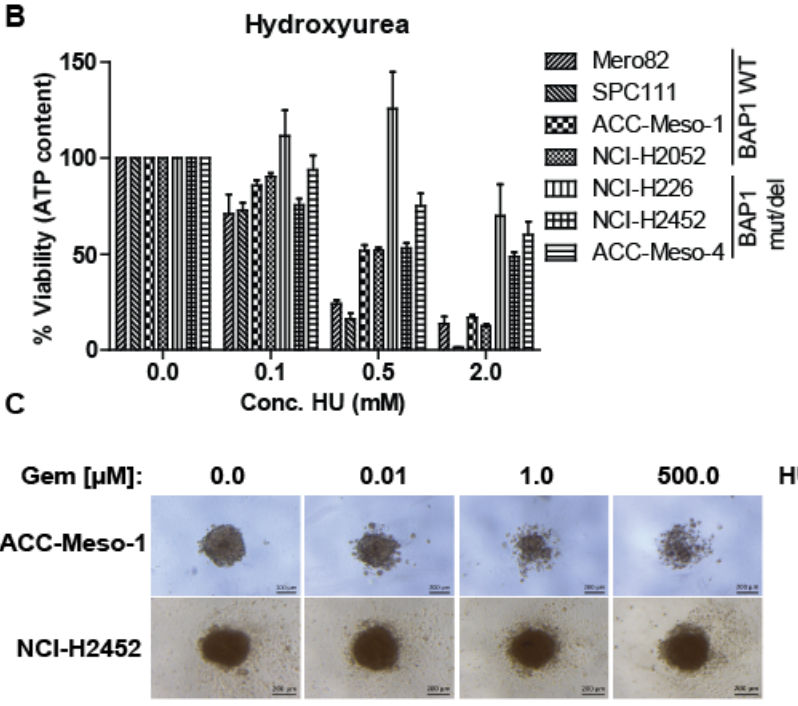
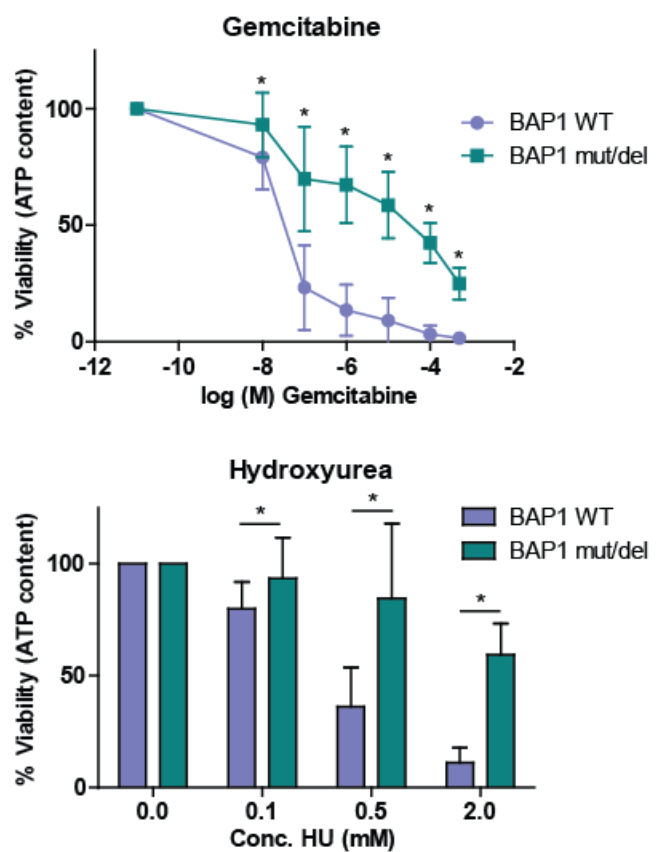

HU [mM]:

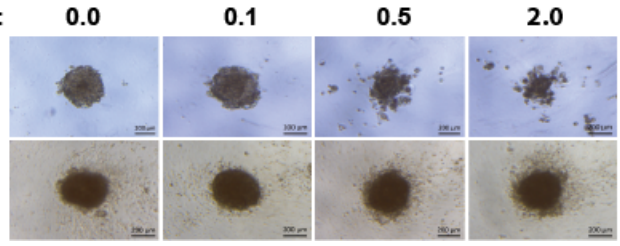

Figure 3. BAP1 proficient MPM cell lines are more sensitive to gemcitabine and hydroxyurea.

Spheroids obtained from BAP1 WT cell lines (NCI-H2052, ACC-Meso-1, Mero82, SPC111) and BAP1 mut/del cell lines (ACC-Meso-4, NCI-H226, NCI-H2452) were treated with: 0.01, 0.1, 1, 10, 100 or $500 \mu \mathrm{M}$ of gemcitabine or $0.1,0.5$ or $2 \mathrm{mM}$ of hydroxyurea or remained untreated. (A) Quantification of ATP content after 6 days of gemcitabine treatment (left panel). Data are presented as mean \pm SEM from $\geq 3$ independent experiments. Pooled means from from BAP1 proficient vs BAP1 deficient cell lines (right panel). (B) Quantification of ATP content after 6 days of hydroxyurea treatment (left panel). Data are presented as mean \pm SEM from $\geq 3$ independent experiments. Pooled means from from BAP1 proficient vs BAP1 deficient cell lines (right panel). Significance was determined by Mann-Whitney U-test $\left({ }^{*} p<0.05\right)$. (C) Representative spheroids treated with either gemcitabine or hydroxyurea. 


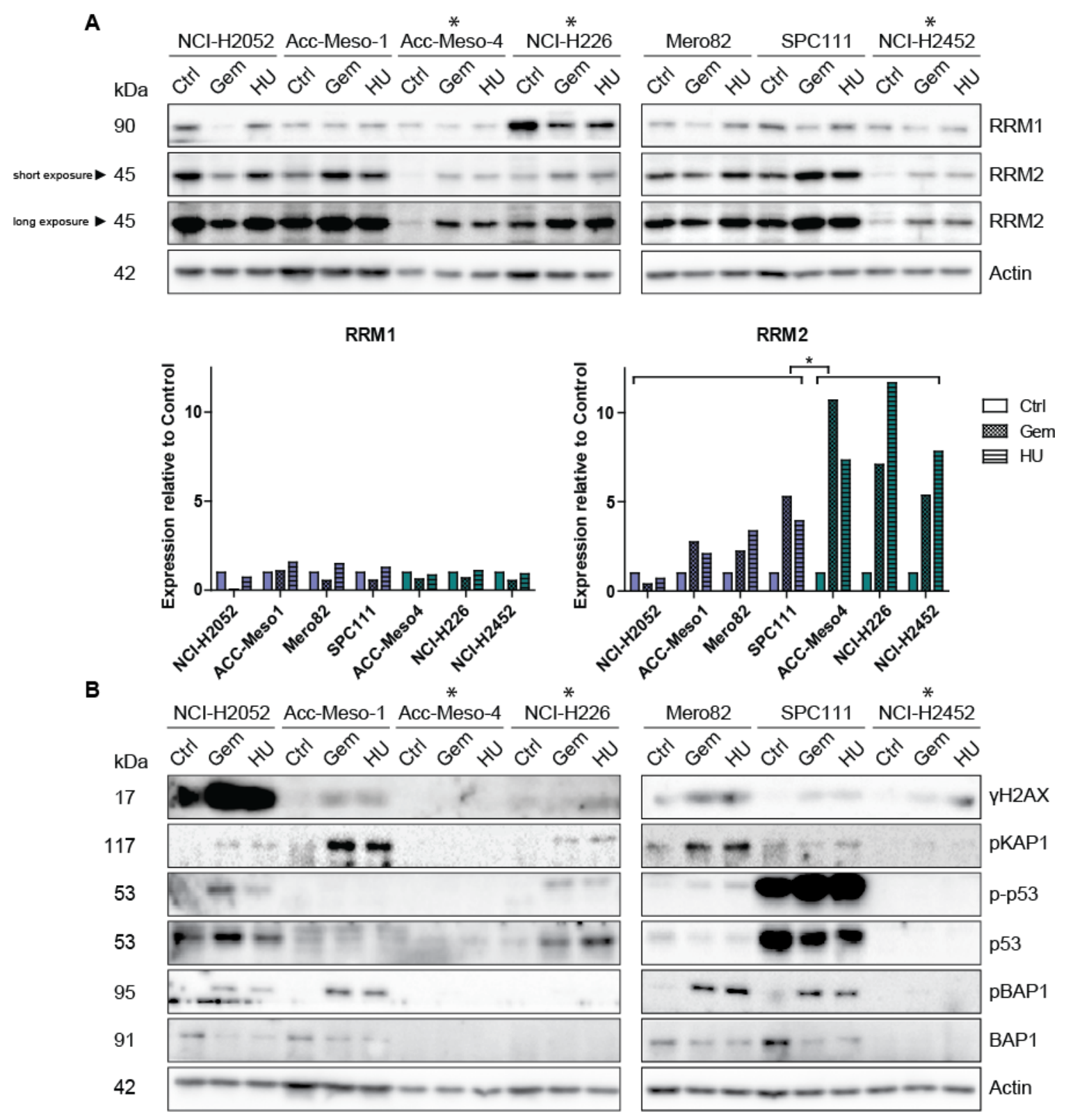

Figure 4. RNR inhibition-induced RRM2 up-regulation is higher in BAP1 mut/del cell lines and

is accompanied by lower levels of residual DNA damage response. Spheroids obtained from BAP1

WT cell lines (NCI-H2052, ACC-Meso-1, Mero82, SPC111) and BAP1 mut/del cell lines (ACCMeso-4, NCI-H226, NCI-H2452 marked with *) were treated with $10 \mu \mathrm{M}$ of gemcitabine or $2 \mathrm{mM}$ of hydroxyurea or remained untreated (Ctrl) and lysed after $48 \mathrm{~h}$. Protein extracts were then analysed by western blotting. (A) Western blot analysis of RRM1 and RRM2 expression and quantification of RRM1 and RRM2 expression was normalised against actin and the data shown are relative to the controls (Ctrl). Representative of two independent experiments. Significance was determined by 
bioRxiv preprint doi: https://doi.org/10.1101/381533; this version posted July 31, 2018. The copyright holder for this preprint (which was

not certified by peer review) is the author/funder, who has granted bioRxiv a license to display the preprint in perpetuity. It is made available under aCC-BY-NC-ND 4.0 International license.

Mann-Whitney U-test $\left({ }^{*} p<0.05\right)$. (B) Expression of DNA damage response markers: yH2AX, pKAP1 phospho-p53 (Ser15, p-p53), total p53 and of phospho-BAP1 (Ser592, pBAP1) and total BAP1. 
A

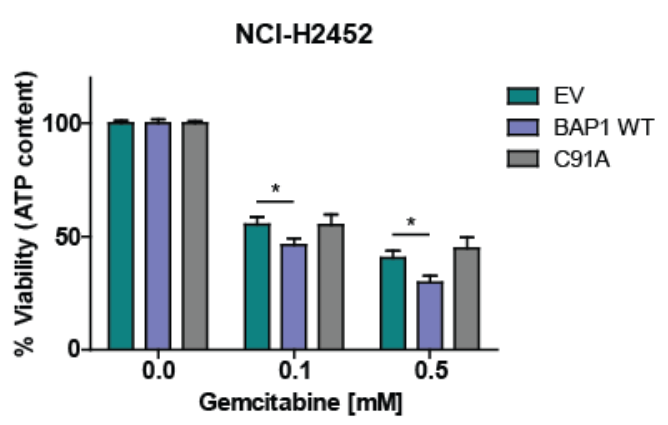

B

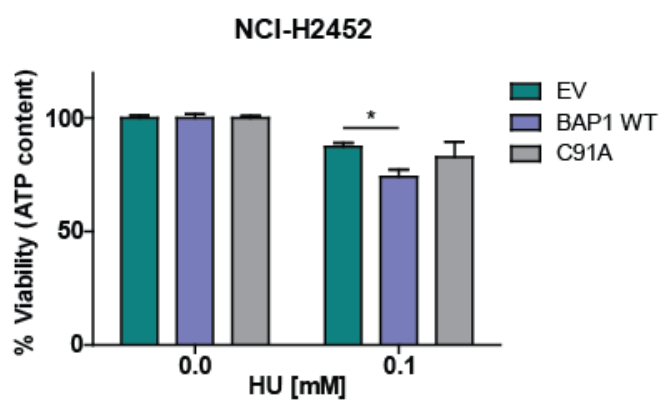

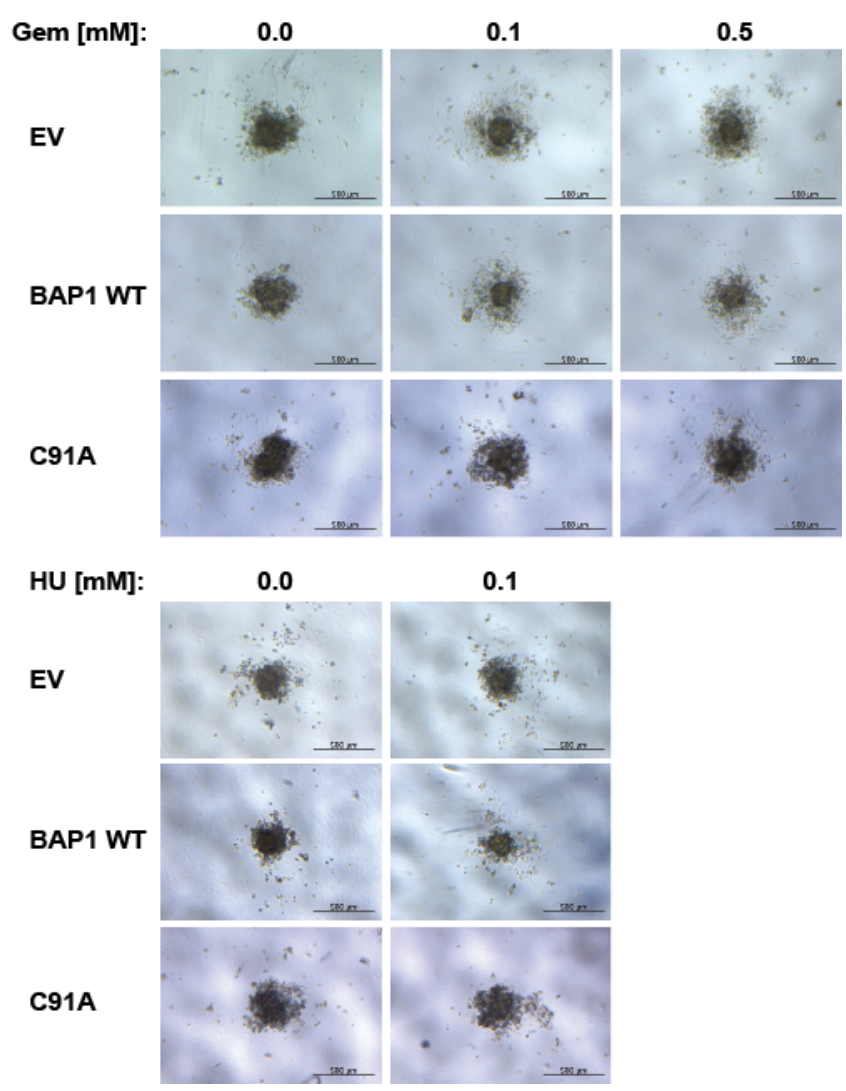

Figure 5. Sensitivity of BAP1 WT MPM cells is dependent on its DUB activity. Spheroids obtained from NCI-H2452 stably expressing either empty vector (EV), BAP1 wild-type (BAP1 WT) or BAP1 C91A mutant (C91A) were treated with 0.1 or $0.5 \mathrm{mM}$ of gemcitabine or $0.1 \mathrm{mM}$ of hydroxyurea or remained untreated. (A) Quantification of the ATP content after 6 days of treatment with gemcitabine relative to the control (left panel) and representative spheroids are presented (right panel). (B) Quantification of the ATP content after 6 days of treatment with hydroxyurea relative to the control (left panel) and representative spheroids are shown (right panel). Data are presented as mean \pm SEM from 3 independent experiments. Significance was determined by Mann-Whitney U-test $\left({ }^{*} p<0.05\right)$. 

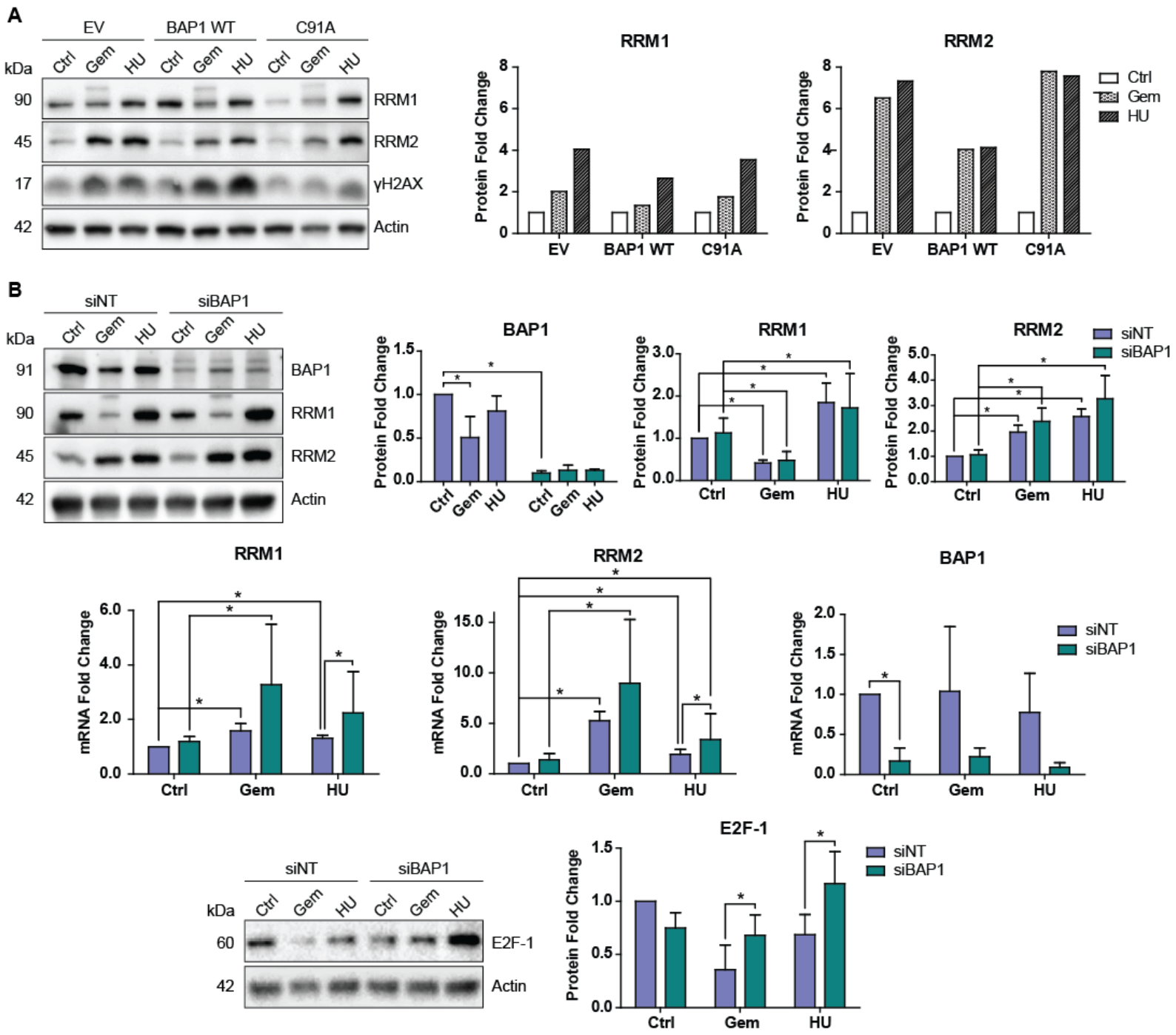

Figure 6. BAP1 regulates RRM2 up-regulation upon RNR inhibition. (A) Spheroids obtained from

NCI-H2452 stably expressing empty vector (EV), BAP1 wild-type (BAP1 WT) or BAP1 C91A mutant (C91A) were treated with $10 \mu \mathrm{M}$ of gemcitabine or $2 \mathrm{mM}$ of hydroxyurea or remained untreated (Ctrl) for $48 \mathrm{~h}$ and lysed. Protein extracts were then analysed by western blotting and probed for RRM1, RRM2 and yH2AX. Western blot (left panel) and western blot quantification of RRM1, RRM2 expression (right panel) normalised against actin and the data shown are relative to the controls (Ctrl) presented as mean from 2 independent experiments. (B) SPC111 (BAP1 WT) cell line was transfected with either siNT or siBAP1 and then treated with $0.1 \mu \mathrm{M}$ of gemcitabine or $0.2 \mathrm{mM}$ of hydroxyurea or remained untreated (Ctrl). Protein and RNA were extracted after $48 \mathrm{~h}$. Representative blot (left upper panel) and a quantification of BAP1, RRM1 and RRM2 (right upper panels) and E2F-1 (bottom panels) expression on the protein level normalised against actin and the data shown are 
relative to the siNon-Targeting untreated cells (siNT Ctrl) presented as mean \pm SD from 4

independent experiments. RRM1, RRM2 and BAP1 expression on mRNA level (middle panels) are relative to the siNon-Targeting untreated cells (siNT Ctrl) presented as means \pm SD from 3

independent experiments. Significance was determined by Student's $t$-test $\left({ }^{*} p<0.05\right)$. 